\title{
ON THE THEORY OF HENSELIAN RINGS, II
}

\author{
MASAYOSHI NAGATA
}

In a previous paper, ${ }^{1)}$ we studied a general theory of integrally closed Henselian integrity domains and some properties of Henselian valuation rings. The present paper is its continuation. The main aim of the present paper is to study a general theory of Henselian local integrity domains; in the present paper we call a ring $\mathfrak{b}$ a local ring if $\mathfrak{D}$ is a quasi-local ring and if the intersection of all powers of the maximal ideal of $D$ is zero, and in this case we introduce a topology by taking the system of all powers of the maximal ideal as a system of neighbourhoods of zero.

Chapter I is concerned mainly with integrally closed local integrity domains: We prove that 1 ) if a Henselian ring $\mathfrak{h}$ contains an integrally closed quasi-local integrity domain $\mathfrak{b}$, then $\mathfrak{h}$ contains the Henselization of $\mathfrak{0}$, provided that the maximal ideal of $\mathfrak{h}$ lies over that of $\mathfrak{b}$ and 2) if $\mathfrak{b}$ is an integrally closed local integrity domain then its Henselization $D^{*}$ is a local ring which contains $D$ as a dense subspace; here, if $D$ is Noetherian then so is $D^{*}$, too.

In Chapter II, we prove first the following: Let $\mathfrak{o}$ be an integrally closed quasi-local integrity domain and let $\mathfrak{D}^{*}$ be its Henselization. If $\mathfrak{p}$ is a prime ideal of $\mathfrak{D}$ then $\mathfrak{p o}^{*}$ is a semi-prime ideal; when $\mathfrak{D} / \mathfrak{p}$ is integrally closed, then $\mathfrak{p o}^{*}$ is a prime ideal. Then we study the nature of $\mathfrak{o}^{*} / \mathfrak{p o}^{*}$ and study some properties of general Henselian integrity domains.

In our treatment we make use of following two lemmas: 1) If an integrity domain $\mathfrak{D}$ is finitely generated (over a prime integrity domain), then for any prime ideal $\overline{\mathfrak{p}}$ of the integral closure $\overline{\mathfrak{D}}$ of $\mathfrak{D}$ in its quotient field, $\overline{D_{\bar{p}}}$ is Noetherian.

2) Let $\mathfrak{D}$ be a Noetherian local integrity domain. If the completion of $\mathfrak{D}$ has no nilpotent elements, then the integral closure $\bar{D}$ of $\mathfrak{D}$ in its quotient field is a finite $\mathfrak{D}$-module. These lemmas will be discussed in Appendix.

Received September 30, 1953.

1) On the theory of Henselian rings, Nagoya Math. J. 5 (1953), pp. 45-57, which will be referred as [H.R.] in the present paper. 


\section{Chapter I. Henselizations of integrally closed local integrity domains}

\section{§1. A lemma on finite module over a ring}

When $\mathfrak{D}$ is a ring, the intersection of all maximal ideal of $\mathfrak{o}$ will be called the $J$-radical of $\mathfrak{D}$. Then it is evident that if $m$ is the $J$-radical of a ring $\mathfrak{D}$, every element $a$ of $\mathrm{o}$ such that $a \equiv 1 \bmod \mathrm{m}$ is a unit in $\mathrm{D}$. Therefore we can show

Lemma $1^{2)} \quad$ Let $\mathrm{o}$ be a ring and let $\mathrm{m}$ be its J-radical. Let $M$ be a finite D-module. If $M \mathrm{~m}=M$, then $M=(0)$.

Proof. Let $u_{1}, \ldots, u_{n}$ be a basis of $M$ over $D_{\text {. The }}$ The have $u_{i}$ $=\sum_{j} a_{i j} u_{j}, a_{i j} \in \mathfrak{m}(1 \leqq i \leqq n)$. Let $d$ be the determinant $\left|\delta_{i j}-a_{i j}\right|$ (where $\delta_{i j}$ denotes the Kronecker $\delta$ ). Then it is evident that $d \equiv 1 \bmod \mathrm{m}$. Therefore $d$ is a unit in $\mathfrak{D}$. On the other hand, it is evident that $d u_{j}=0$ for each $j$, whence $u_{j}=0$ for each $j$. Thus we see our assertion.

Corollary. Let $\mathrm{nl}$ and $\mathrm{D}$ be the same as above. Let $M$ be a finite $\mathfrak{D}$-module. If $N$ is a sub-0-module of $M$ such that $N+M \mathrm{~m}=M$, then $M=N$.

Proof. Set $\bar{M}=M / N$. Then $\bar{M} \mathrm{~m}=\bar{M}$ and $\bar{M}$ is a finite $\mathrm{o}$-module. Therefore $\bar{M}=(0)$, that is, $M=N$.

\section{§ 2. Quasi-decompositional extensions}

Definition. A decompositional extension $\mathfrak{D}^{\prime}$ of an integrally closed integrity domain $\mathfrak{D}$ with respect to its prime ideal $\mathfrak{p}$ is an integrally closed integral extension of $\mathfrak{D}$ such that there exists a separable normal extension $\bar{D}$ of $\mathfrak{D}$ in which there exists a prime ideal $\bar{p}$ such that $\mathfrak{D}^{\prime}$ becomes the decomposition ring of $\bar{p}$ with respect to $\mathfrak{D}$ and $\bar{p} \cap \mathfrak{D}=\mathfrak{p}$. The prime ideal $\bar{p} \cap \mathfrak{D}^{\prime}$ is called a characteristic prime ideal of this extension.

Definition. Let $\mathfrak{D}$ be an integrally closed quasi-local integrity domain with maximal ideal $\mathfrak{p}$. An integral extension $\mathfrak{S}$ of $\mathfrak{D}$ is called a quasi-decompositional extension of $\mathrm{b}$, if $\mathfrak{Z}$ is obtained by adjoining an element a which is a root of an irreducible monic polynomial $x^{r}+d_{1} x^{r-1}+\ldots+d_{r}$ over $\mathfrak{p}$ and if $d_{r}$ is in $\mathfrak{p}$ and $d_{r-1}$ is not in p. Such an element $a$ is called a characteristic element of this extension and the maximal ideal $(\mathfrak{p}, a) \mathfrak{I}$ is called the characteristic prime

2) This lemma and the corollary were proved in Nagata, On the structure of complete local rings, Nagoya Math. J. 1 (1950). 
ideal of this extension.

Remark. Since $0=(a \bmod (p, a) \Im)$ is a simple root of $x^{r}+d_{1} x^{r-1}+\ldots$ $+d_{r} \bmod \mathfrak{p}, a$ is a simple root of $x^{r}+d_{1} x^{r-1}+\ldots+d_{r}$. Therefore we see that this polynomial $x^{r}+d_{1} x^{r-1}+\ldots+d_{r}$ is separable.

Now we prove

TheOREM 1. Let o be an integrally closed quasi-local integrity domain with maximal ideal $\mathfrak{p}$. Assume that $\mathfrak{S}=\mathrm{o}[a]$ is a quasi-decompositional extension of $\mathfrak{0}$ with characteristic element a. Set $\mathfrak{m}=(\mathfrak{p}, a) \mathfrak{I}$. Then $\mathfrak{I}_{\mathfrak{m}}$ is integrally closed. If moreover $\mathfrak{D}$ is a local ring, then $\Im_{\mathfrak{m}}$ is a local ring and contains $\mathfrak{D}$ as a dense subspace.

Proof. (1) We first assume that $D$ is a local ring and we will prove that $\Im_{\mathfrak{m}}$ is a local ring and contains $\mathcal{D}$ as a dense subspace: It is evident that $\mathfrak{J} / \mathrm{m}$ $=\mathfrak{p} / \mathrm{p}$. Let $f(x)=x^{r}+d_{1} x^{r-1}+\ldots+d_{r}$ be the irreducible monic polynomial over $\mathfrak{D}$ which has $a$ as a root. Set $a^{\prime}=-\left(a^{r-1}+d_{1} a^{r-2}+\ldots+d_{r-1}\right)$. Then since $d_{r-1} \notin \mathfrak{p}, a \in \mathrm{m}$, we see that $a^{\prime}$ is a unit in $3 \mathfrak{m}$. Since $d_{r} \in \mathfrak{p}$, we see that $a$ is in $\mathfrak{p} \mathfrak{I}_{\mathfrak{m}}$, which shows that $\mathfrak{p} \Im_{\mathfrak{m}}=\mathfrak{m} \Im_{\mathfrak{m}}$. Next we show that $\mathfrak{m}^{k} \Im_{\mathfrak{m}} \cap \mathfrak{o} \subseteq \mathfrak{p}^{k}$ for any natural number $k$. Let $b$ be an arbitrary element of $\mathrm{m}^{k} \Im_{\mathfrak{m}} \cap \mathrm{o}$. Since

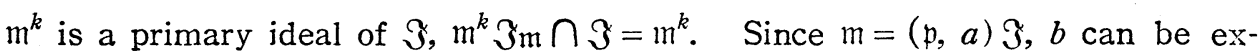
pressed as follows: $b=b_{0}+b_{1} a+\ldots+b_{u} a^{u}, b_{i} \in \mathfrak{p}^{k-i}$ if $k-i>0, b_{k}, \ldots$, $b_{u} \in \mathfrak{0}$. Since $a a^{\prime}=d_{r} \in \mathfrak{p}$, we can write $b a^{\prime u}=e_{0}+e_{1} a+\ldots+e_{r-1} a^{r-1}\left(e_{i} \in \mathfrak{p}^{k}\right)$. Then $\operatorname{spur}\left(b a^{\prime u}\right)=\sum_{i} \operatorname{spur}\left(e_{i} a^{i}\right) \in \mathfrak{p}^{k}$. On the other hand, $\operatorname{spur}\left(b a^{\prime \prime}\right)$ $=b\left(\operatorname{spur}\left(a^{\prime}\right)\right)$. Therefore if we show that $\operatorname{spur}\left({a^{\prime \prime}}^{\prime \prime}\right)$ is not in $\mathfrak{p}$, we complete to show that $\mathfrak{m}^{k} \Im_{\mathfrak{m}} \cap \mathfrak{o} \subseteq \mathfrak{p}^{k}$. Thus we will show that $\operatorname{spur}\left(\boldsymbol{a}^{\prime \prime}\right)$ is not in $\mathfrak{p}$ : Let $a=a_{1}, a_{2}, \ldots, a_{r}$ be the totality of roots of $f(x)$. Then it is evident that $a^{\prime}$ $=a_{2} \cdots a_{r}$. Let $\overline{\mathrm{D}}$ be an almost finite separable normal extension of 0 containing $a$. Let $\overline{\mathfrak{p}}$ be a maximal ideal of $\overline{\mathfrak{D}}$ which lies over $\mathfrak{m}$. Then $a$ is in $\overline{\mathfrak{p}}$ and $a^{\prime}=a_{2} \cdots a_{r}$ is not in $\bar{p}$. Now, $\operatorname{spur}\left({a^{\prime}}^{u}\right)=\sum_{i} a_{1}^{u} \cdots a_{i-1}^{u} a_{i+1}^{u} \cdots a_{r}^{u}$, whence $\operatorname{spur}\left(a^{\prime u}\right) \equiv a_{2}^{u} \cdots a_{r}^{u}=a^{\prime \prime} \bmod \mathfrak{p}^{\prime}$. Therefore $\operatorname{spur}\left(a^{\prime \prime}\right)$ is not in $\mathfrak{p}^{\prime}$, hence it is not in $\mathfrak{p}$. Thus we see that $\mathrm{m}^{k} \mathfrak{\jmath m} \cap \mathfrak{v} \subseteq \mathfrak{p}^{k}$. Since the inverse inclusion $\mathrm{m}^{k} \mathfrak{Y}_{\mathfrak{m}}$ $\cap \mathfrak{o} \supseteqq \mathfrak{p}^{k}$ is evident, we see that $\mathfrak{m}^{k} \Im_{\mathfrak{m}} \cap \mathfrak{o}=\mathfrak{p}^{k}$ for any natural number $k^{3)}$ Since $\bigcap_{k} \mathfrak{p}^{k}=(0)$ and since $\mathfrak{I}_{\mathfrak{m}}$ is algebraic over $\mathfrak{D}$, we see also that $\bigcap_{k} \mathfrak{m}^{k} \Im_{\mathfrak{m}}=(0)$,

3) This equality holds in general without assumption that $D$ is a local ring. 
which shows that $\Im_{\mathfrak{m}}$ is a local ring. Further, it follows easily that $\mathfrak{D}$ is a dense subspace of $\Im_{\mathfrak{m}}$ from $\Im_{\mathfrak{m}} / \mathfrak{m} \Im_{\mathfrak{m}}=\Im / \mathfrak{m}=\mathfrak{v} / \mathfrak{p}$ and $\mathfrak{m}^{k} \Im_{\mathfrak{m}} \cap \mathfrak{D}=\mathfrak{p}^{k}$ (for any k).

(2) Let $\mathfrak{b}^{\prime}$ be the integral closure of $\mathfrak{Y}$ in its quotient field. We show that $m \mathrm{o}^{\prime}$ has only one prime divisor: Let $\overline{\mathfrak{D}}$ be again an almost finite separable normal extension of $\mathfrak{v}$ containing $\mathfrak{v}^{\prime}$ and let $\overline{\mathrm{m}}_{1}, \ldots, \overline{\mathrm{m}}_{t}$ be the totality of prime divisors of $\bar{m} \overline{\mathfrak{p}}$. We denote by $G$ the Galois group of $\overline{\mathfrak{D}}$ over $\mathfrak{D}$ and by $H$ the subgroup of $G$ which corresponds to $\mathfrak{D}^{\prime}$. Then it is evident that if $\sigma \in G$ and if $\sigma \notin H$ then $\overline{\mathrm{m}}^{\sigma}$ contains $a^{\sigma}(\neq a)$ and therefore $\overline{\mathrm{m}}_{i}^{\sigma}$ is not any of $\overline{\mathrm{m}}_{j}$. Therefore if $\sigma \in G$ and if $\overline{\mathfrak{m}}_{i}^{\sigma}$ is one of $\overline{\mathfrak{m}}_{j}$, then $\sigma \in H$. Since there exists an element $\sigma$ of $G$ such that $\overline{\mathfrak{m}}_{i}^{\sigma}=\overline{\mathfrak{m}}_{j}$ for any given pair $(i, j), \overline{\mathrm{m}}_{1}, \ldots, \overline{\mathrm{m}}_{t}$ are transitively transposed by elements of $H$. Therefore $\overline{\mathfrak{m}}_{1} \cap \mathfrak{v}^{\prime}=\overline{\mathrm{m}}_{j} \cap \mathfrak{o}^{\prime}$ for any $j$, because $H$ is the Galois group of $\overline{0}$ over $\mathrm{D}^{\prime}$. This proves that $\mathrm{mo}^{\prime}$ is contained in only one maximal ideal $\mathfrak{p}^{\prime}=\overline{\mathfrak{m}}_{1} \cap \mathfrak{d}^{\prime}$.

(3) We prove our assertion when $\mathfrak{D}$ is Noetherian: With the same notation as in (2), we have only to show that $\mathfrak{o}_{\mathfrak{p}^{\prime}}^{\prime}=\Im_{\mathfrak{m}}$, by virtue of (1). Since $\mathfrak{D}^{\prime}$ is separable over $\mathfrak{D}$ and since $\mathfrak{D}$ is Noetherian, it is evident that $\mathfrak{D}^{\prime}$ is a finite D-module. Let $S$ be the complementary set of $m$ with respect to $\Im$. Then since $m \mathfrak{o}^{\prime}$ has the unique prime divisor $\mathfrak{p}^{\prime}, \mathrm{o}_{\mathrm{s}}^{\prime}$ is a local ring with maximal ideal $\mathfrak{p}^{\prime} \mathfrak{b}_{s}^{\prime}$, whence $\mathfrak{o}_{\mathfrak{p}}^{\prime}=\mathfrak{o}_{s^{\prime}}^{\prime}$. Therefore we see that $\mathfrak{o}_{\mathfrak{p}}^{\prime}$ is a finite $\Im_{\mathfrak{m}}$-module.

$(3, a)$ When $\mathfrak{D}^{\prime}$ is a decompositional extension of $\mathfrak{D}$ with characteristic prime ideal $\mathfrak{p}^{\prime}$ : By Theorem 1 in [H.R.], we see that $\mathfrak{p}^{\prime} \mathfrak{o}_{\mathfrak{p}^{\prime}}^{\prime}=\mathfrak{p} \mathfrak{o}_{\mathfrak{p}^{\prime}}^{\prime}$ and $\mathfrak{o}_{\mathfrak{y}^{\prime}}^{\prime} / \mathfrak{p o}_{\mathfrak{p}^{\prime}}^{\prime}=\mathfrak{v} / \mathfrak{p}$ $=\mathfrak{I}_{\mathfrak{m}} / \mathfrak{m} \Im_{\mathfrak{m}}$. Therefore we have that $\mathfrak{m o}_{\mathfrak{p}^{\prime}}^{\prime}+\mathfrak{I}_{\mathfrak{m}}=\mathfrak{o}_{\mathfrak{p}^{\prime}}^{\prime}$. Since $\mathfrak{o}_{\mathfrak{p}^{\prime}}^{\prime}$ is a finite $\mathfrak{I}_{\mathfrak{m}}$ module, we see that $\mathfrak{o}_{\mathfrak{p}^{\prime}}^{\prime}=\mathfrak{I}_{\mathfrak{m}}$ by virtue of Lemma 1 .

$(3, b)$ Now we treat the general case (under the assumption that $\mathfrak{D}$ is Noetherian). We use the same notations as in (2). Let $\widetilde{\mathfrak{b}}$ be the decomposition ring of $\overline{\mathfrak{m}}_{1}{ }^{4}$ ) Then by $(3, a)$ above, we see that $\widetilde{\mathfrak{D}} \tilde{\mathfrak{m}}$ (where $\tilde{\mathfrak{m}}=\widetilde{\mathfrak{D}} \cap \overline{\mathfrak{m}}_{1}$ ) contains $\mathfrak{D}$ and $\mathfrak{D}_{\mathfrak{p}}^{\prime}$ as dense, subspaces, which shows that $\mathfrak{o}_{\mathfrak{p}}^{\prime}$ contains $\mathfrak{D}$ as a dense subspace. Therefore we see that $m_{\mathfrak{p}^{\prime}}^{\prime}=\mathfrak{p}_{\mathfrak{p}^{\prime}}^{\prime}=\mathfrak{p}^{\prime} \mathfrak{o}_{\mathfrak{p}^{\prime}}^{\prime}$. Thus we see also in this case that $\mathfrak{m o v}_{\mathfrak{p}}^{\prime}+\Im_{\mathfrak{m}}=\mathfrak{o}_{\mathfrak{p}^{\prime}}^{\prime}$, and we see that $\mathfrak{c}_{\mathfrak{p}^{\prime}}^{\prime}$ coincides with $\Im_{\mathfrak{m}}$.

(4) Now we prove our theorem in general case. By virtue of (1) above, we have only to show that $\Im_{\mathfrak{m}}$ is integrally closed. Let $b / c \quad(b, c \in \Im, c \neq 0)$

4) In this case, the decomposition ring $\widetilde{o}$ of $\overline{\mathfrak{m}} 1$ over $\mathfrak{D}$ is the decomposition ring of $\overline{\mathfrak{n}}_{11}$ over $b^{\prime}$ as will be easily seen from arguments in our proof in (2). 
be an element of the quotient field of $\Im$ which is integral over $\Im_{m}:(b / c)^{n}$ $+c_{1}(b / c)^{n-1}+\ldots+c_{n}=0\left(c_{i} \in \Im_{\mathfrak{m}}\right)$. Choose an element $d$ of $\Im$ which is not in $\mathrm{m}$ so that $c_{i} d \in \mathfrak{Y}$ for any $i$. Considering $b d / c$ instead of $b / c$, we may assume that $c_{1}, \ldots, c_{n}$ are in $\Im$. Then we can write $b=t_{0}+t_{1} a+\cdots+t_{r-1} a^{r-1}$, $c=u_{0}+u_{1} a+\ldots+u_{r-1} a^{r-1}, c_{i}=v_{i, 0}+v_{i, 1} a+\ldots+v_{i, r-1} a^{r-1} \quad\left(t_{j}, u_{j}, v_{i, j} \in \mathfrak{D}\right)$. Let $\Im^{\prime}$ be the subring of $\mathfrak{D}$ generated by $1, t_{0}, \ldots, t_{r-1}, u_{0}, \ldots, u_{r-1}, v_{1,0}$, $\ldots, v_{1, r-1}, v_{2,0}, \ldots, v_{n, r-1}$, and let $\mathfrak{J}^{\prime \prime}$ be the integral closure of $\mathfrak{Y}^{\prime}$ in its quotient field. Set $m^{\prime \prime}=\mathfrak{p} \cap \mathfrak{Y}^{\prime \prime}$ and consider $\mathfrak{J}^{*}=\mathfrak{J}^{\prime \prime} \mathfrak{m}^{\prime \prime}$. Then since $\mathfrak{Y}^{\prime}$ is finitely generated, $\Im^{*}$ is Noetherian (for the proof, see Appendix $\S 2$ ). There-

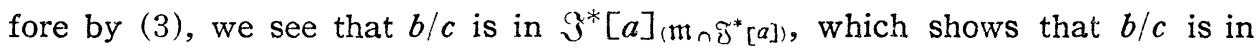
$\Im \mathfrak{m}$. Therefore $\Im_{\mathfrak{m}}$ is integrally closed and our proof is completed.

COROLlary 1. Let $\mathfrak{o}$ be an integrally closed quasi-local integrity domain with maximal ideal $\mathrm{p}$. Assume that $\mathrm{D}^{\prime}$ is an almost finite decompositional extension of o with respect to $\mathfrak{p}$. Let $\mathfrak{p}^{\prime}$ be a characteristic prime ideal and let a be an element of $\mathfrak{p}^{\prime}$ which is in none of maximal ideals of $\mathfrak{b}^{\prime}$ other than $\mathfrak{p}^{\prime}$. Set 3 $=\mathrm{o}[a], \mathfrak{m}=\mathfrak{p}^{\prime} \cap \mathfrak{Y}_{\text {. Then }}$ we have $\mathfrak{c}^{\prime}=\mathfrak{M} \mathrm{m}$. When, in this case, $\mathfrak{o}$ is a local ring, then $\mathrm{o}^{\prime}$ is a local ring and contains o as a dense subspace.

Corollary 2. Let $\mathfrak{D}, \mathfrak{p}, \mathfrak{D}^{\prime}$ and $\mathfrak{p}^{\prime}$ be the same as in Corollary 1. Let $a^{\prime}$ be an element of $\mathfrak{D}^{\prime}$ which is not in $\mathfrak{p}^{\prime}$ and is in every maximal ideal of $\mathfrak{b}^{\prime}$ other than $\mathfrak{p}^{\prime}$. Set $\Im^{\prime}=\mathfrak{o}\left[a^{\prime}\right]$ and $\mathfrak{m}^{\prime}=\mathfrak{p}^{\prime} \cap \mathfrak{\Im}^{\prime}$. Then $\mathfrak{o}_{\mathfrak{p}^{\prime}}^{\prime}=\mathfrak{S}_{\mathfrak{m}^{\prime}}^{\prime}$.

Proof. Set $a=a^{\prime}-\operatorname{spur}\left(a^{\prime}\right)$. Then it is evident that this a satisfies the condition of $a$ in Corollary 1.

\section{§ 3. Henselizations of integrally closed local integrity domains}

Theorem 2. Let $\mathrm{D}$ be an integrally closed quasi-local integrity domain with maximal ideal $\mathfrak{p}$ and let $\mathfrak{h}$ be $a$ Henselian ring which contains $\mathfrak{D}$ as a subring. If the maximal ideal $\mathfrak{M}$ of $\mathfrak{h}$ lies over $\mathfrak{p}$ (i.e., $\mathfrak{M} \cap \mathfrak{D}=\mathfrak{p}$ ), then $\mathfrak{h}$ contains the Henselization $\mathfrak{D}^{*}$ of $\mathfrak{D}$ ( $u p$ to an isomorphism over $\left.\mathfrak{b}\right)$. When $\mathfrak{D}$ is a local ring, $\mathfrak{D}$ is a dense subspace of $\mathrm{D}^{*}$.

Proof. Let $\mathfrak{f}$ be the totality of pairs $(\mathfrak{r}, \sigma)$ of subrings $\mathfrak{r}$ of $\mathfrak{h}$ and isomorphisms $\sigma$ such as 1) $\mathfrak{r}$ is an integrally closed quasi-local integrity domain (and $\mathfrak{M}$ lies over the maximal ideal of $\mathfrak{r}), 2$ ) $\sigma$ is an isomorphism from $\mathfrak{r}$ into the Henselization $0^{*}$ of $\mathfrak{o}$. In $f$ we introduce a partial order as follows: $\left(\mathfrak{r}_{1}, \sigma_{1}\right)$ $\leqq\left(\mathfrak{r}_{2}, \sigma_{2}\right)$ if and only if $\left.i\right) \mathfrak{r}_{1}$ is a subring of $\mathfrak{r}_{2}$ and ii) $\sigma_{1}$ is the restriction of 
$\sigma_{2}$ in $r_{1}{ }^{5)} \quad$ Then as is easily seen, $f$ is an inductive set. Therefore by Zorn's lemma, we see easily that there exists a maximal member $\left(\mathfrak{r}^{\prime}, \sigma^{\prime}\right)$ in $\mathfrak{f}$. If $\mathfrak{r}^{\prime}$ is not Henselian, then we have easily a contradiction by virtue of Theorem 1 . Therefore $\mathfrak{r}^{\prime}$ must be the Henselization of $\mathfrak{b}$. Now we assume that $\mathrm{p}$ is a local ring. Let $\mathfrak{f}^{\prime}$ be the subset of $\mathfrak{f}$ which is consisted all of $(\mathfrak{r}, \sigma)$ such that $\mathfrak{r}$ contains $\mathfrak{D}$ as a dense subspace. Then it will be also easy to see that $f^{\prime}$ is also inductive. Therefore there exists a maximal member $\left(\mathfrak{r}^{\prime \prime}, \sigma^{\prime \prime}\right)$ in $\mathfrak{f}^{\prime}$. If $\mathfrak{r}^{\prime \prime}$ is not Henselian, we have easily a contradiction by Theorem 1 , whence $\mathfrak{r}^{\prime \prime}$ must be also the Henselization of $\mathfrak{b}$, which shows that the Henselization of $\mathfrak{b}$ contains $\mathfrak{b}$ as a dense subspace.

COROllary. Let $\mathrm{o}$ be an integrally closed local integrity domain with maximal ideal $\mathfrak{p}$. If $\mathfrak{b}^{\prime}$ is a decompositional extension of $\mathfrak{o}$ with respect to $\mathfrak{p}$ and with characteristic prime ideal $\mathfrak{p}^{\prime}$, then $\mathfrak{D}^{\prime}$, is a local ring and contains $\mathfrak{D}$ as a dense subspace.

Proof is easy if we observe that the Henselization of $n_{p^{\prime}}^{\prime}$ is the Henselization of $\mathrm{D}$.

THEOREM 3. If $\mathrm{D}$ is an integrally closed Noetherian local integrity domain, then the Henselization $\mathrm{D}^{*}$ of $\mathrm{D}$ is Noetherian (and contains $\mathrm{D}$ as a dense subspace).

Proof. Let $\bar{D}$ be the completion of $\mathfrak{D}$. Then we may consider $\overline{\mathfrak{D}}$ also as the completion of $0^{*}$, by Theorem 2. Since $\overline{\mathfrak{D}}$ is Noetherian, we have only to show that if $a$ is an ideal of $0^{*}$ which has a finite basis then $a \overline{0} \cap 0^{*}=a$. Let $a_{1}, \ldots$, $a_{n}$ be a basis of $a$ and let $b$ be an element of $n \overline{0} \cap 0^{*}$. We may assume that $a_{1}, \ldots, a_{n}$ and $b$ are integral over $\mathrm{o}$. Then we can find a finite decompositional extension $\mathfrak{D}^{\prime}$ of $\mathfrak{D}$ with respect to the maximal ideal $\mathfrak{p}$ of $\mathrm{n}$, such that $a_{1}$, $\ldots, a_{n}, b$ are in $\mathfrak{o}^{\prime}$ and that $\mathfrak{p}^{\prime}=\mathfrak{p o}^{* *} \cap \mathrm{o}^{\prime}$ is a characteristic prime ideal of this extension. Then by Theorem 2 we see that $\mathfrak{r}_{\mathfrak{p}}^{\prime}$ is contained in $\mathfrak{D}^{*}$ as a dense subspace. Therefore $\overline{\mathrm{D}}$ is also the completion of $\mathfrak{o}^{\prime}$. Since $\mathfrak{o}_{\mathfrak{p}}^{\prime}$ is Noetherian, $\left(a_{1}, \ldots, a_{n}\right) \overline{\mathrm{D}} \cap \mathrm{o}_{\mathfrak{F}^{\prime}}^{\prime}=\left(a_{1}, \ldots, a_{n}\right) \mathfrak{o}_{\mathfrak{p}^{\prime}}^{\prime}$. Therefore $b \in\left(a_{1}, \ldots, a_{n}\right) \mathrm{o}_{\mathrm{p}^{\prime}}^{\prime}$, which shows that $b \in a \overline{0} \cap \mathfrak{o}^{*}$. Therefore $\bar{a} \overline{0} \cap \mathfrak{o}^{*}=a$.

By virtue of this theorem, when we consider completions of integrally closed Noetherian local integrity domains, we may consider the completions of the Henselizations of the given local integrity domains. In this sense, it will

5) It will be not hard to see that $(r, \sigma) \leqq\left(r^{\prime}, \sigma^{\prime}\right)$ if $\mathfrak{r} \leqq \mathrm{r}^{\prime}$. 
be important to consider a problem that under what condition Henselian local integrity domain is algebraically closed in its completion. ${ }^{6)}$ Here we will prove some lemmas concerning algebraically closedness of Henselian local integrity domains in their completions:

Definition. Let $\mathfrak{o}$ be a local integrity domain. Then: (1) We say that $\mathfrak{n}$ is algebraically closed in its completion $\bar{D}$ if $\overline{\mathcal{D}}$ is an integrity domain and if every element $a$ of $\bar{D}$ which is algebraic over $\mathfrak{D}$ is already in $0 . \quad$ (2) We say that $\mathfrak{D}$ is separably algebraically closed (or purely inseparably algebraically closed) in its completion $\bar{D}$ if $\bar{D}$ is an integrity domain and if every element $a$ of $\bar{D}$ which is separably algebraic (or purely inseparably algebraic) over $\mathfrak{D}$ is already in $\mathrm{D}$.

Lemma 2. Let o be a Noetherian Henselian local integrity domain. If $\mathrm{o}$ is algebraically closed (or separably algebraically closed or purely inseparably algebraically closed) in its completion, then so is the integral closure $\mathfrak{D}^{\prime}$ of $\mathfrak{D}$ in its quotient field and $\mathrm{D}^{\prime}$ is a finite $\mathrm{D}$-module. Conversely, if $\mathrm{o}^{\prime}$ is a finite $\mathrm{D}$-module and if $\mathrm{D}^{\prime}$ is algebraically closed (or separably algebraically closed or purely inseparably algebraically closed) in its completion, then so is $\mathrm{D}$, too.

Proof. Assume that $\mathfrak{D}$ is algebraically closed in its completion $\overline{0}$. Then it is easy to see that $D^{\prime}$ is a finite 0 -module (for the proof, see Appendix, $\S 1$ ). Assume that an element $a$ of the completion $\overline{\mathfrak{l}}^{\prime}$ of $\mathfrak{b}^{\prime}$ is algebraic over $\mathfrak{D}^{\prime}$. Let $d$ be an element of $\mathfrak{o}$ such that $d 0^{\prime} \cong \mathfrak{o}(d \neq 0)$. Then $d a$ is algebraic over $\mathfrak{D}$ and is in $\bar{D}$. Therefore we see that $d a$ is in $\mathfrak{D}$ and therefore $a$ is in the quotient field $L$ of $\mathrm{D}$. Since $\mathrm{o}^{\prime}=L \cap \overline{\mathrm{D}}^{\prime}$ (for the proof, see Appendix, $\S 1$ ), we see that $a$ is in $\mathfrak{D}^{\prime}$. Thus we see that $\mathfrak{D}^{\prime}$ is algebraically closed in $\bar{D}^{\prime} .{ }^{i)}$ Conversely, assume that $\mathfrak{D}^{\prime}$ is a finite $\mathfrak{D}$-module and that $\mathfrak{D}^{\prime}$ is algebraically closed in its completion $\overline{\mathcal{D}}^{\prime}$. Since $\overline{\mathrm{D}} \subseteq \overline{\mathrm{D}}^{\prime}, \overline{\mathrm{D}}$ is an integrity domain. If an element $a$ of $\overline{\mathrm{D}}$ is algebraic over $\mathrm{n}$, then $a$ is algebraic over $\mathrm{D}^{\prime}$ and therefore $a$ is in $\mathrm{D}^{\prime}$, hence $a$ is in $L$. Since $\mathfrak{D}=L \cap \overline{\mathrm{D}}$, we see that $a$ is in $\mathfrak{D}$. Separable case or purely inseparable case can be treated similarly.

Lemma 3. Let o be a Noetherian Henselian integrity domain. Then $\mathrm{o}$ is

6) As was shown in [H.R.], there exists a valuation ring which is not algebraically closed in its completion. Some sufficient conditions for our protlem will be shown in a latter paper.

i) Since $d \overline{\mathfrak{v}}^{\prime} \subseteq \overline{\mathfrak{D}}$, that $\overline{\mathrm{v}}^{\prime}$ is an integrity domain is evident. 
algebraically closed (or separably algebraically closed or purely inseparably algebraically closed) if and only if for any finite integral (or separably integral or purly inseparably integral) extension $\mathrm{D}^{\prime}$ of $\mathrm{o}$ such that $\mathrm{D}^{\prime}=\mathrm{D}[a]$ by an element a of $\mathrm{D}^{\prime}$, the completion of $\mathrm{v}^{\prime}$ is an integrity domain.

Proof. Assume that $\mathfrak{D}$ is algebraicaliy closed in its completion. Let $\mathfrak{D}^{\prime \prime}$ be the integral closure of $\mathrm{D}$ in its quotient field. Then by Lemma $2, \mathfrak{D}^{\prime \prime}$ is algebraically closed in its completion $\bar{D}^{\prime \prime}$. Let $f(x)$ be the irreducible monic polynomial over $\mathfrak{D}^{\prime \prime}$ which has $a$ as a root. Then since $\mathfrak{b}^{\prime \prime}$ is algebraically closed in $\overline{\mathfrak{D}}^{\prime \prime}, f(x)$ is irreducible over the quotient field of $\bar{D}^{\prime \prime}$. Therefore the completion of $\mathfrak{D}^{\prime \prime}[a]$ is an integrity domain, whence so is the completion of $\mathrm{D}[a]=\mathrm{D}^{\prime}$. Conversely, assume that $D$ is not algebraically closed in its completion $\bar{D}$. Then there exists an element $a$ of $\bar{D}$ which is integral over $D$ and is not in $\mathrm{D}$, when $\overline{\mathrm{D}}$ is an integrity domain. Then the completion of $o[a]$ is not an integrity domain. For the case when $\bar{D}$ is not an integrity domain, our assertion is evident. Separable case or purely inseparable case are settled similarly.

COROLlary 1. If a Noetherian Henselian integrity domain $\mathrm{D}$ is algebraically closed (or separably algebraically closed) in its completion, then so is any separable finite integral extension of $\mathrm{D}$.

Corollary 2. Assume that a Noetherian Henselian integrity domain $\mathfrak{D}$ is algebraically closed in its completion. If $\mathrm{o}$ is of characteristic zero, then any finite integral extension of $\mathrm{D}$ is algebraically closed in its completion.

Corollary 3. Assume that a Noetherian local integrity domain $\mathfrak{0}$ is separably algebraically closed in its completion. If $\mathrm{D}^{\prime}$ is a finite integral extension of $\mathrm{n}$, then the zero ideal of the completion of $\mathrm{D}^{\prime}$ is a primary ideal.

COROllary 4. If a class $\$$ of Noetherian local integrity domains satisfies the follouing three conditions, then every member of $\mathbb{B}$ is algebraically closed in its completion:

1) If $\mathfrak{o}$ is a member of $\left(\mathbb{S}\right.$, then there exists a local integrity domain $\mathfrak{D}^{\prime}$ which is contained in $\mathfrak{D}$ such that $\mathrm{i}) \mathrm{D}$ is a finite $\mathfrak{D}^{\prime}$-module and ii) $\mathfrak{D}^{\prime}$ is algebraically closed in its completion.

2) If $\mathfrak{D}$ is a member of $\mathbb{B}$, then every almost finite integral extension of $\mathfrak{D}$ is a finite $\mathrm{D}-\mathrm{module}$ and is in $\mathbb{B}$.

3) If $\mathrm{D}$ is a member of $\mathbb{B}$ and if $\mathfrak{p}$ is a prime ideal of $\mathrm{D}$, then $\mathrm{D} / \mathrm{p}$ is in $\mathbb{B}$. 
Proof. Our condition 2) and 3) shows that for every member 0 of (3) the completion of o has no nilpotent element. ${ }^{8)}$ On the other hand, by Corollary 3 and by condition 1), we see that the zero ideal of the completion of 0 is a primary ideal. Thus we see that the completion of $D$ is an integrity domain. This holds for any member of (S), whence by Lemma 3 and by condition 2), we see that $\boldsymbol{D}$ is algebraically closed in its completion.

\section{Chapter II. General Henselian integrity domain}

\section{§4. Preliminaries}

Leмma 4. Let o be an integrally closed quasi-local integrity domain with maximal ideal $p$ and let $\mathrm{q}$ be a prime ideal of $\mathrm{n}$. Assume that an integral extension $\}=\mathfrak{n}[a]$ is a quasi-decompositional extension of 0 with respect to $p$ and with characteristic element $a$. Set $\mathrm{m}=(\mathfrak{p}, a) 3$ and $3^{*}=3 \mathrm{mt}$. If $\mathrm{q}^{*}$ is a prime divisor of $\mathrm{q}^{*}$, then $\mathrm{q}^{*} \cap \mathrm{D}=\mathrm{q}$.

Proof. Assume the contrary. Then we can find an element $b$ of $o$ which is not in $q$ and an element $c$ of $3^{*}$ which is not in $q 3^{*}$ such that $b c \in q 3^{*}$. We can find an element $c^{\prime}$ of 3 which is not in $m$ so that $c c^{\prime} \in \mathcal{G}, b c c^{\prime} \in q 3$. Then we can write $\left.c c^{\prime}=c_{0}+c_{1} a+\ldots+c_{r-1} a^{r-1}\left(c_{i} \in \mathrm{o}, r=[\}: 0\right]\right)$. Since $1, a, \ldots$, $a^{r-1}$ are linearly independent over $\left.0, b c c^{\prime} \in q\right\}$ shows $b c_{\imath} \in a$ for every $i$. Since $\mathfrak{q}$ is prime, $c_{i} \in \mathfrak{q}$ for every $i$ and therefore $\left.c c^{\prime} \in \mathfrak{q}\right\}$ which is a contradiction.

Now let $n$ be again an integrally closed quasi-local integrity domain with maximal ideal $\mathfrak{p}$ and let $q$ be a prime ideal of $n$. Let $\bar{b}$ be an almost finite separable normal extension of $\mathfrak{D}$ with Galois group $G$ and let $\mathfrak{q}$ be a prime ideal of $\overline{\mathrm{D}}$ such that $\overline{\mathfrak{q}} \cap \mathrm{D}=q$. Further let $\overline{\mathfrak{p}}$ be a maximal ideal of $\overline{\mathrm{D}}$ which contains $\bar{\eta}_{0}$ Let $H_{\mathfrak{p}}$ and $H_{\mathfrak{q}}$ be the decomposition groups of $\overline{\mathfrak{b}}$ and $\overline{\mathfrak{q}}$ respectively and let $H$ be the subgroup generated by $H_{\mathfrak{p}}$ and $H_{\mathfrak{q}}$. Let $\mathrm{D}^{\prime}$ be the integrally closed integral extension of $\mathfrak{o}$ which corresponds to $H$. Set $p^{\prime}=\bar{p} \cap \mathfrak{o}^{\prime}, q^{\prime}=\bar{q} \cap \mathfrak{o}^{\prime}$ and $\mathfrak{o}^{\prime \prime}$ $=q_{\mathfrak{p}^{\prime}}^{\prime}$ and let $S$ be the complementary set of $q$ with respect to $n$. Let $b$ be an element of $\mathfrak{p}^{\prime}$ which is in none of maximal ideals of $\mathfrak{p}^{\prime}$ other than $\mathfrak{p}^{\prime}$ and set $\mathfrak{Y}^{\prime}=\mathfrak{b}[b], \mathrm{m}^{\prime}=\mathfrak{p}^{\prime} \cap \mathfrak{Y}^{\prime}$. Then by Corollary 1 to Theorem 1 we see that $\mathfrak{o}^{\prime \prime}=\mathfrak{S}^{\prime} \mathrm{\prime n}^{\prime}$. Therefore it is evident, by virtue of Theorem 1 in [H.R.] and its corollary, that

Lemma $5 . \quad \mathrm{o}^{\prime} / \mathfrak{q}^{\prime}$ is an integral extension of $\mathfrak{o} / \mathfrak{q}$ and has the same quotient field with $\mathrm{b} / \mathrm{q}$. Therefore there exists an element $c b-d(c, d \in 0)$ such that $c b-d$

8) See Theorem 4 in Nagata, Some remarks on local rings, Nagoya Math. J. 6 (1953). 
$\in \mathfrak{q}^{\prime}, c \notin \mathfrak{q}$ and $(\mathfrak{q}, c b-d) \mathrm{o}_{s}^{\prime \prime}=\mathfrak{q}^{\prime} \mathrm{o}_{s}^{\prime \prime}$.

Now let $\widetilde{\mathfrak{D}}$ be the decomposition ring of $\overline{\mathfrak{p}}$ and set $\widetilde{\mathfrak{p}}=\overline{\mathfrak{p}} \cap \tilde{\mathfrak{n}}, \tilde{\mathfrak{q}}=\overline{\mathfrak{q}} \cap \tilde{\mathfrak{n}}$.

LEMMA 6. Let a be an element of $\tilde{\mathfrak{p}}$ which is in none of maximal ideals of $\widetilde{n}$ other than $\tilde{\mathfrak{p}}$. Let $f(x)=x^{r}+d_{1} x^{r-1}+\ldots+d_{r}$ be the irreducible monic polynomial over $\mathrm{D}^{\prime}$ having $a$ as a root. Then $\bar{f}(x)=\left(f(x) \bmod \mathrm{q}^{\prime}\right)$ is irreducible over the quotient field $L$ of $\mathfrak{b}^{\prime} / \mathfrak{q}^{\prime}$. Therefore $\mathfrak{q}^{\prime} \tilde{\mathfrak{D}}_{\mathfrak{p}}$ is a prime ideal.

Proof. By our choice of $\mathfrak{b}^{\prime}, \mathfrak{b}^{\prime}$ is the intersection of the decomposition ring of $\bar{p}$ and that of $\bar{q}$. Assume that there exist prime ideals of $\widetilde{\mathfrak{d}}$ which lie over $q^{\prime}$ other than $\tilde{q}$; let $\tilde{q}_{1}, \ldots, \tilde{q}_{s}$ be the totality of them. Let $c$ be an element of $\tilde{q}$ which is in none of $\tilde{q}_{j}$. Then it is evident that $c$ is contained in both decomposition rings of $\bar{p}$ and $\bar{q}$, which is a contradiction. Therefore $\tilde{\mathfrak{q}}$ is the unique prime ideal of $\tilde{\mathfrak{o}}$ which lies over $q^{\prime}$. Therefore $\bar{f}(x)=\bar{g}(x)^{e}$ with an irreducible polynomial $\bar{g}(x)$ over $\bar{L}$. On the other hand, by our choice of $a$, ( $a \bmod$ $\left.q^{\prime}\right)$ is a simple root of $\bar{f}(x)$. Therefore $e=1$, which shows that $\bar{f}(x)$ is irreducible over $\bar{L}$.

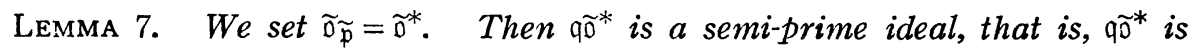
the intersection of all prime ideals of $\widetilde{\mathfrak{D}}^{*}$ which lie over $q$.

Proof. Let the prime divisors of $q^{*}$ be $q_{1}^{*}, \ldots, q_{t}^{*}$. Then by Lemma 4 they lie over $q$. Therefore we see that $q \widetilde{D}_{S}^{*} \cap \widetilde{D}^{*}=q \tilde{D}^{*}$ (with the same $S$ as above). Since $\mathfrak{v}^{\prime}$ is contained in the decompositional extension of $\overline{\mathfrak{q}}$, we see that $\mathfrak{q}^{\prime}$ is the primary component of $q d^{\prime}$ belonging to $q^{\prime}$ (see our proof of Theorem 1 ). Therefore by virtue of Lemma 6 , we see that each $q_{i}^{*}$ is the primary component of $q \widetilde{o}^{*}$ belonging $q_{i}^{*}$. We consider $\widetilde{\mathrm{D}}_{S}^{*} / \mathfrak{q}^{*}$. By Lemma 5 we see that $\mathfrak{q}_{i}^{*} \widetilde{\mathfrak{D}}_{S}^{*} / \mathfrak{q}_{s}^{*}$ is generated by an element. Therefore $\tilde{\mathbb{D}}_{S}^{*} / q \tilde{\mathrm{d}}_{S}^{*}$ is a Noetherian ring, and therefore $u \widetilde{\mathcal{D}}_{s}^{*}=\mathrm{q}_{1}^{*} \widetilde{\mathfrak{D}}_{S}^{*} \cap \cdots \cap \mathrm{q}_{t}^{*} \widetilde{\mathfrak{D}}_{S}^{*}$, whence $\mathrm{q}^{*} \tilde{\mathrm{D}}^{*}=\mathrm{q}_{1}^{*} \cap \cdots \cap \mathrm{q}_{t}^{*}$.

\section{§. Prime ideals and Henselizations}

In the present paragraph, let 0 be an integrally closed quasi-local integrity domain and let $D^{*}$ be the Henselization of $\mathrm{n}$. Further let $p$ be the maximal ideal of $\mathfrak{b}$ and let $q$ be a prime ideal of $\mathfrak{b}$.

THEOREM 4. $\mathrm{q0}^{*}$ is the intersection of all prime ideals of $\mathrm{D}^{*}$ which lie over $\mathrm{q}$.

Proof. Let $q^{*}$ be a prime divisor of $q 0^{*}$. If $q^{*} \cap 0 \neq q$, then taking an element $a$ of $a^{*} \cap 0$ which is not in $a$ and an element $b$ of $b^{*}$ which is not in $q 0^{*}$ 
such that $a b \in q 2^{*}$, we have a con rradiction easily by Lemma 4 . Therefore $\mathfrak{q}^{*} \cap \mathfrak{v}=\mathfrak{q}$. Let $\mathfrak{a}^{*}$ be the intersection of all prime ideals of $\mathfrak{o}^{*}$ which lie over q. Then we see that $a^{*} \supseteqq q 0^{*}$. Assume that $a^{*} \neq 40^{*}$. Then taking an element $c$ of $a^{*}$ which is not in $q \mathcal{J}^{*}$, we have easily a contradiction by Lemma 7. Thus we see our assertion.

THEOREM 5. If $\mathrm{q}^{*}$ is a prime divisor of $\mathrm{q0}^{*}$, then $\mathrm{o}^{*} / \mathrm{qo}^{*}$ is minimal among Henselian integrity domains which contain $\mathrm{o} / \mathrm{q}$ and whose maximal ideal lie over $\mathfrak{p} / \mathfrak{q}$. Further if an Henselian integrity domain $\mathfrak{h}$ contains $\mathfrak{b} / \mathfrak{q}$ and if its maximal ideal lies over $\mathfrak{p} / \mathfrak{q}$, then $\mathfrak{h}$ contains a Henselian ring which is isomorphic to $\mathfrak{b}^{*} / \mathfrak{q}^{*}$ with a suitable prime divisor $\mathrm{q}^{*}$ of $\mathrm{qo}^{*}$.

Proof. We first prove the last assertion. Let $f$ be the totality of pairs $(\mathfrak{r}, \sigma)$ of subrings $\mathfrak{r}$ of $D^{*}$ containing $\mathfrak{D}$ and homomorphisms $\sigma$ such that 1$) \mathfrak{r}$ is an integrally closed quasi-local integrity domain (and $\mathbf{p o}^{*}$ lies over the maximal ideal of $\mathfrak{r}$ ) and 2) $\sigma$ is an homomorphism from $\mathfrak{r}$ into $\mathfrak{h}$ under which $\mathfrak{D} / \mathfrak{p}$ is mapped identically. Then by the same way as in the proof of Theorem 2, we introduce a partial order in $\mathfrak{f}$. Then it is easy to see that $f$ becomes an inductive set. Therefore there exists a maximal member $\left(\mathfrak{r}^{\prime}, \sigma^{\prime}\right)$ of $\mathfrak{f}$. If $\mathfrak{r}^{\prime}$ is not Henselian, then we can find a quasi-decompositional extension $\mathfrak{r}^{\prime}[a]$ of $\mathfrak{r}^{\prime}$ with a characteristic element $a$. Let $f(x)$ be the irreducible monic polynomial over 0 having $a$ as a root. Then since $\mathfrak{h}$ is Henselian, $\bar{f}(x)=\sigma^{\prime}(f(x))$ has a linear factor $x-\bar{a}$ (and $\bar{a}$ is in the maximal ideal of $\mathfrak{h}$ ). Therefore there exists a homomorphism $\sigma^{\prime \prime}$ from $\mathfrak{r}^{\prime}[a]$ onto $\sigma^{\prime}\left(\mathfrak{r}^{\prime}\right)[\bar{a}]$ such that $\sigma^{\prime}$ is the restriction of $\sigma^{\prime \prime}$ in $\mathfrak{r}^{\prime}$ and $\sigma^{\prime \prime}(a)$ $=\bar{a}$. Since $\mathfrak{h}$ is quasi-local and since $\bar{a}$ is in the maximal ideal of $\mathfrak{h}$, we may consider that $\sigma^{\prime \prime}$ is an homomorphism from $\mathfrak{r}^{\prime}[a]_{\mathfrak{m}^{\prime}}$ into $\mathfrak{h}$, where $\mathfrak{m}^{\prime}$ denotes the maximal ideal of $\mathfrak{r}^{\prime}[a]$ containing $a$. By theorem $1, \mathfrak{r}^{\prime}[a] \mathfrak{m}^{\prime}$ is integrally closed, which is a contradiction to our choice of $\left(\mathfrak{r}^{\prime}, \sigma^{\prime}\right)$. Therefore $\mathfrak{r}^{\prime}$ must be Henselian, which shows our last assertion. Assume that $D^{*} / q^{*}$ is not minimal. Then there exists a Henselian ring $\mathfrak{h}$ which is contained properly in $0^{*} / \mathfrak{q}^{*}$ and whose maximal ideal lies over $\mathfrak{p} / \mathfrak{q}$. Then by above proof, we see that $\mathfrak{h}$ contains $\mathfrak{o}^{*} / \mathfrak{q}_{1}^{*}$ by a suitable prime divisor $q_{1}^{*}$ of $q 0^{*}$. Therefore a contradiction will be easily shown by the following

THEOREM 6. There is a one to one correspondense between maximal ideals of the integral closure $\mathfrak{D}^{\prime}$ of $\mathfrak{D} / \mathfrak{q}$ in its quotient field and the prime divisors of 
$\mathrm{qo}^{*}$ : If a maximal ideal $\mathfrak{p}^{\prime}$ of $\mathrm{b}^{\prime}$ and a prime divisor $\mathrm{q}^{*}$ of $\mathrm{q0}^{*}$ correspond to each other, then the integral closure of $\mathrm{o}^{*} / \mathrm{q}^{*}$ in its quotient field is the Henselization of $\mathfrak{o}_{\mathfrak{p}}^{\prime}$.

Proof. Let $\overline{\mathrm{D}}$. be the totality of separably algebraic element over $\mathfrak{D}$ (in an algebraically closed field containing $D$ ) and let $G$ be the Galois group of $\overline{0}$ over D. Let $\bar{p}$ be a maximal ideal of $\bar{D}$. Let $\widetilde{D}$ be the decomposition ring $\bar{p}$ and set $\tilde{\mathfrak{p}}=\overline{\mathfrak{p}} \cap \tilde{\mathfrak{D}}$. Then we may assume that $\mathfrak{o}^{*}=\widetilde{\mathfrak{D}} \tilde{\mathfrak{p}}$. On the other hand let $\overline{\mathfrak{q}}$ be a prime ideal of $\overline{\mathfrak{o}}$ which lies over $\mathfrak{q}$. Let $H_{\mathfrak{q}}$ and $H_{\mathfrak{p}}$ be the decomposition groups of $\bar{q}$ and $\bar{p}$ respectively. Now we will show the following lemmas:

Lemma 8. The Galois group of $\overline{\mathrm{D}} / \overrightarrow{\mathrm{q}}$ over $\mathfrak{D}^{\prime}$ is a homomorphic image of $H_{\mathfrak{q}}$.

Proof. It is evident that every element of $H_{\mathfrak{q}}$ induces an automorphism of $\bar{b} / \bar{q}$ over $\mathfrak{D}^{\prime}$. For the converse, considering $D_{\mathfrak{q}}$ instead of $\mathfrak{D}$, we may assume that $q$ is maximal. Then by the same way as in the case of algebraic integers, we can prove our lemma.

Lemma 9. The totality of maximal ideals of $\overline{0}$ which contain $\overline{\mathrm{q}}$ and whose residues modulo $\overline{\mathfrak{q}}$ contains the maximal ideal $\mathfrak{p}^{\prime}$ of $\mathfrak{b}^{\prime}$ is $\left\langle\bar{p}^{\left.H_{\mathfrak{p}}{ }^{\circ H_{\mathfrak{q}}}\right\}}\right.$ with an element $\sigma$ of $G$.

Proof is easy by virtue of Lemma 8 and by Lemma 1 in [H.R.].

On the other hand, it is evident also that

Lemma 10. Let $\mathrm{q}^{*}$ be a prime divisor of $\mathrm{qo}^{*}$ and set $\tilde{\mathrm{q}}=\mathrm{q}^{*} \cap \tilde{\mathrm{o}}$. Then the totality of prime ideals of $\overline{\mathfrak{D}}$ which are contained in $\overline{\mathfrak{p}}$ and lie over $\tilde{\mathfrak{q}}$ is $\left\{\overline{\mathfrak{q}}^{H} \mathcal{q}^{\tau H_{\mathfrak{p}}}\right\}$ with an element $\tau$ of $G$.

Now we will proceed our proof of Theorem 6: By Lemma 9, we can give a one to one correspondence between maximal ideals $\mathfrak{p}^{\prime}$ of $\mathfrak{b}^{\prime}$ and two-sided classes $H_{\mathfrak{p}} \sigma H_{\mathfrak{q}}$ of $G$ as is given there. We take a maximal ideal $\mathfrak{p}_{0}^{\prime}$ of $\mathfrak{p}^{\prime}$. Then by a rechoice of $\bar{p}$, we may assume that $\mathfrak{p}_{0}^{\prime}$ corresponds to $H_{\mathfrak{p}} H_{\mathfrak{q}}$ (and that $\overline{\mathfrak{p}}$ contains $\bar{q}$ ). Now by Lemma 10 , we can find a one to one correspondence between prime divisors of $q^{*}{ }^{*}$ and two-sided classes $H_{\mathfrak{q}}=H_{\mathfrak{p}}$ of $G$ as is given there. Since we can give a one to one correspondence between two-sided classes $H_{p} \sigma H_{q}$ and two-sided classes $H_{\mathfrak{q}} \tau H_{\mathfrak{p}}$ such that $H_{\mathfrak{p} \sigma} H_{\mathfrak{q}}$ corresponds to $H_{\mathfrak{q}} \sigma^{-1} H_{\mathfrak{p}}$, we can give a one to one correspondence between maximal ideals of $\mathfrak{b}^{\prime}$ and prime divisors of $q 0^{*}$. Assume that a maximal ideal $\mathfrak{p}^{\prime}$ of $\mathfrak{b}^{\prime}$ corresponds to a prime di- 
visor $\mathfrak{q}^{*}$ of $q 0^{*}$ by the correspondence given above. Let $H_{\mathfrak{p}} \sigma H_{\mathfrak{q}}$ be the two-sided class which corresponds to $\mathfrak{p}^{\prime}$.

1) When $H_{\mathfrak{p}} \sigma H_{\mathfrak{q}}=H_{\mathfrak{p}} H_{\mathfrak{q}}$. In this case $\mathfrak{p}^{\prime}=\mathfrak{p}_{0}^{\prime}$ by our choice. Set $\tilde{\mathfrak{q}}=\overline{\mathfrak{q}} \cap \tilde{\mathfrak{o}}$. Then it is evident that $q^{*}=\widetilde{q} \mathfrak{D}^{*}$. On the other hand, it is evident that the decomposition group of $\bar{p} / \bar{q}$ is the homomorphic image of $H_{\mathfrak{q}} \cap H_{\mathfrak{p}}$ by the natural mapping, whence the Henselization of $o_{p^{\prime}}^{\prime}$ contains $D^{*} / q^{*}$ and has the same quotient field with $\mathfrak{o}^{*} / \mathfrak{q}^{*}$. It is evident that the Henselization of $\mathfrak{o}^{\prime}$, is integral over $\mathfrak{D}^{*} / \mathfrak{q}^{*}$, whence we see our assertion in this case.

2) Now we show our assertion in general case. Considering $\bar{p}^{s}$ instead of $\bar{p}$, we construct the Henselization of $0^{*}$ by the decomposition ring of $\bar{p}^{\sigma}$. Then the same holds for this case if we consider $\bar{q}^{\sigma^{-1}}$ instead of $\bar{q}$. Therefore we see our assertion also in this case.

Corollary. If $\mathrm{o} / \mathfrak{q}$ is integrally closed, then $\mathrm{q}^{*}$ is a prime ideal and $\mathrm{o}^{*} / \mathrm{q}^{*}$ is the Henselization of $\mathrm{o} / \mathrm{q}$ and therefore, in this case, $\mathrm{D}^{*} / \mathrm{q}^{*}$ is integrally closed.

Remark. This corollary can be proved directly by Lemma 6 and the following

Lemma 11. With the same notations as in Lemma 5 , if $\mathrm{o}^{\prime} / \mathrm{q}^{\prime}=\mathrm{o} / \mathfrak{q}$, then $\mathrm{qo}^{\prime \prime}$ is a prime ideal.

Proof. Since $\mathfrak{v}^{\prime} / \mathfrak{q}^{\prime}=\mathfrak{d} / \mathfrak{q}$, we may assume that the element $b$, which we chose in Lemma 5 , is in $q^{\prime}$. Let $f(x)=x^{r}+d_{1} x^{r-1}+\ldots+d_{r}$ be the irreducible monic polynomial over $\mathfrak{o}$ which has $b$ as a root. Then $d_{r}$ is in $\mathfrak{q}$ and $d_{r-1}$ is not in $\mathfrak{p}$. Set $b^{\prime}=b^{r-1}+d_{1} b^{r-2}+\ldots+d_{r-1}$. Then $b^{\prime}$ is not in $\mathrm{m}$, therefore $q \mathfrak{d}^{\prime \prime}$ contains $b$. Thus we see that $q 0^{\prime \prime}$ is a prime ideal.

Next we show

THEOREM 7. A quasi-local integrity domain $\mathfrak{h}$ is Henselian if and only if every integral extension of $\mathfrak{h}$ is quasi-local.

Proof. It is easy that if $\mathfrak{h}$ is Henselian, then every its integral extension is quasi-local by virtue of Theorem 6. ${ }^{9)} \quad$ Therefore we will show the converse part of our assertion. Let $b_{1}$ be an integrally closed quasi-local integrity domain which has a prime ideal $q_{1}$ such that $\mathfrak{b}_{1} / q_{1}=\mathfrak{h}$. Let $\mathrm{o}^{*}$ be the Henselization of

9) Making no use of Theorem 6, we can prove this directly; see, G. Azumaya, On maximally central algebras, Nagoya Math. J. 2 (1950). 
$\mathfrak{D}_{1}$. Since the integral closure $\overline{\mathfrak{h}}$ of $\mathfrak{h}$ in its quotient field is Henselian, we can find a prime divisor $\mathfrak{q}^{*}$ of $\mathfrak{D}^{*}$ such that $\mathfrak{D}^{*} / \mathfrak{q}^{*}$ is contained in $\overline{\mathfrak{h}}$. Let $\mathfrak{o}$ be a quasilocal integrity domain which is maximal among integrally closed quasi-local rings such that 1 ) they are contained in $0^{*}, 2$ ) they contains $D_{1}$ and 3 ) the residue class rings of them modulo $q^{* 10)}$ coincides with $\mathfrak{h}$. (Exsistence of such $\mathfrak{0}$ will be easy by virtue of Zorn's lemma). Now it is sufficient to show that 0 is Henselian, because every homomorphic image of Henselian ring is also Henselian. Assume that $\mathfrak{D}$ is not Henselian. Then we can find a quasi-decompositional extension $\mathrm{o}[a]$ of $\mathrm{D}$ with a characteristic element $a$ (which is not in $\mathrm{D}$ ). Let $\mathfrak{D}^{\prime}$ be the integral closure of $\mathrm{o}[a]$ in its quotient field and set $\mathfrak{p}^{\prime}=\mathfrak{p}^{*} \cap \mathfrak{o}^{\prime}$, where $\mathfrak{p}^{*}$ is the maximal ideal of $\mathfrak{b}^{*}$. Further set $\mathfrak{q}^{\prime}=\mathfrak{q}^{*} \cap \mathfrak{o}^{\prime}$. Then since $\mathfrak{b}^{\prime} / q^{\prime}$ is an integral extension of $\mathfrak{h}, \mathfrak{b}^{\prime} / \mathfrak{q}^{\prime}$ is quasi-local, which shows that if we denote by $\mathfrak{a}^{\prime}$ the intersection of all maximal ideals of $\mathfrak{b}^{\prime}$ other than $\mathfrak{p}^{\prime}$, then $\left(\mathfrak{a}^{\prime}, \mathfrak{q}^{\prime}\right) \mathfrak{d}^{\prime}=\mathfrak{v}^{\prime}$. Therefore we can find an element $b$ of $q^{\prime}$ which is in none of maximal ideals of $\mathfrak{D}^{\prime}$ other than $\mathfrak{p}^{\prime}$. Then it is evident that $\mathrm{D}[b]$ is a quasi-decompositional extension of $\mathfrak{D}$ with a characteristic element $b$ and that $b$ is not in $\mathfrak{D}$. Therefore we may assume that $b=a$. Then it is evident that $\mathrm{D}[a] / \mathfrak{q}^{*} \cap \mathrm{D}[a]=\mathfrak{h}$. Therefore we see that $\mathfrak{o}_{\mathfrak{p}^{\prime}}^{\prime} / \mathfrak{q}^{*} \cap \mathrm{o}_{\mathfrak{p}^{\prime}}^{\prime}=\mathfrak{h}$, because $\mathfrak{o}_{\mathfrak{p}^{\prime}}^{\prime}=\mathrm{o}[a]_{\mathfrak{m}}$ with $\mathfrak{m}=\mathfrak{p}^{\prime} \cap \mathrm{o}[a]$ by virtue of Theorem 1. Since $\mathfrak{o}_{\mathfrak{p}^{\prime}}^{\prime}$ is integrally closed and is contained in $\mathfrak{b}^{*}$, we have a contradiction. Thus we see that $\mathfrak{D}$ is Henselian and therefore we see our assertion.

\section{§. Supplimentary remarks}

I) We may define the Henselization of a quasi-local integrity domain $\mathfrak{b}$ as follows: Let $D^{\prime}$ be an integrally closed quasi-local integrity domain with a prime ideal $q$ such that $\mathfrak{b}^{\prime} / \mathfrak{q}=\mathfrak{v}$. Let $\mathfrak{o}^{*}$ be the Henselization of $\mathfrak{b}^{\prime}$. Then we call $\mathfrak{o}^{*} / \mathfrak{q}^{*}$ the Henselization of $D$.

We can prove easily the uniqueness of the Henselization in this sense.

II) Theorem 5 shows in particular the following assertion, which will be useful to the theory of local rings:

With the same $0, q, b^{*}$ as in $\S 5$, if there exists an integrally closed quasilocal integrity domain $\mathfrak{b}^{\prime}$ such that $\mathfrak{D} / \mathfrak{q}$ is a ring of quotients of a finite integral extension of $\mathfrak{D}^{\prime}$ with respect to its maximal ideal, then $\mathfrak{D}^{*} / \mathfrak{q}^{*}$ is a finite module

10) This means "modulo the ideal which is the intersection of $q^{*}$ with the ring of consideration." 
over the Henselization of $b^{\prime}$, where $q^{*}$ is a prime divisor of $q 0^{*}$.

III) Lemma 2 in [H.R.] can be generalized as follows:

LeMma A. Let $\mathrm{n}$ be an integrally closed integrity domain with a maximal ideal $\mathfrak{p}$. Assume that $\mathfrak{D}^{\prime}$ is an almost finite separable integral extension of $\mathrm{D}$ which is integrally closed. Let $\mathfrak{p}_{1}^{\prime}, \ldots, p_{h}^{\prime}$ be the totality of maximal ideals of $\mathfrak{D}^{\prime}$ which lie over $\mathfrak{p}$. If an element $a$ of $\mathfrak{b}^{\prime}$ is not in $\mathfrak{p}_{1}^{\prime}$ and is in every one of $\mathfrak{p}_{2}^{\prime}, \ldots, \mathfrak{p}_{h}^{\prime}$, then a satisfies an irreducible monic polynomial $f(x)=x^{r}+d_{1} x^{r-1}+\ldots+d_{r}$ such that $d_{1} \notin \mathfrak{p}, d_{2}, \ldots, d_{r} \in \mathfrak{p}$.

Proof. Let $\overline{\mathfrak{D}}$ be an almost finite separable normal extension of $\mathfrak{D}$ containing $\mathrm{D}^{\prime}$. Let $G$ be the Galois group of $\overline{\mathrm{D}}$ and let $H$ be the subgroup of $G$ which corresponds to $\mathfrak{D}^{\prime}$. Let $\bar{p}$ be a maximal ideal of $\bar{D}$ which lies over $\mathfrak{p}_{1}^{\prime}$. Then $\left\{\bar{p}^{H}\right\}$ is the totality of maximal ideals of $\overline{0}$ which lie over $p_{1}^{\prime}$. We may assume that the quotient field of $\mathrm{o}^{\prime}$ is generated by $a$ over the quotient field of $\mathrm{b}$. Then $a^{\rho}=a(\sigma \in G)$ if and only if $\sigma \in H$. Therefore any conjugate of $a$ which is different from $a$ is in $\bar{p}$. Therefore we see our assertion easily.

Lemma B. Let $\mathfrak{p}, \mathfrak{p}, \mathfrak{o}^{\prime}, \mathfrak{p}_{1}^{\prime}, \ldots, \mathfrak{p}_{h}^{\prime}$ be the same as in Lemma $A$ above. If an element $b$ of $p_{1}^{\prime}$ is in none of $p_{2}^{\prime}, \ldots, p_{h}^{\prime}$, then $b$ satisfies an irreducible monic polynomial $f(x)=x^{r}+e_{1} x^{r-1}+\ldots+e_{r}$ over o such that $e_{r} \in \mathfrak{p}$ and $e_{r-1} \notin \mathfrak{p}$.

Proof is similar to that of Lemma A.

COROLlary. With the same notations as above, $a$ and $b$ are in the decomposition ring of $\bar{p}$.

IV) Here the writer wishes to add two conjectures on completions of Henselian local integrity domains:

Conjecture 1. If 0 is a Noetherian Henselian integrity domain, then the zero ideal of the completion of $\mathfrak{D}$ is a primary ideal.

Conjecture 2. There exists a Noetherian integrally closed Henselian integrity domain such that its completion is not an integrity domain.

Remark. Conjecture 1 has much relation with the following problem:

"Let $\mathfrak{b}$ be a Noetherian local integrity domain. Then does the number of maximal ideals of the integral closure of $D$ in its quotient field coincide to the number of prime divisors of zero of the completion of $D$ ? Has the zero ideal 
of the completion of $\mathfrak{D}$ no imbedded prime divisor ?"

As for the second conjecture, it is equivalent to the following problem:

"Is there exists an integrally closed Noetherian local integrity domain whose completion is not an integrity domain?"

\section{Appendix}

\section{$\S 1$. Finiteness of integral closure}

Proposition 1. Let $\mathfrak{o}$ be a Noetherian complete local integrity domain. Then the integral closure of $D$ in its quotient field is a finite $D$-module.

Since this is an assertion properly concerned with the theory of local rings, the writer wishes to write a proof of this assertion in a latter paper on local rings. ${ }^{11}$ Therefore we omit the proof in the present paper.

Lemma 12. Let $\mathrm{o}$ be a Noetherian local integrity domain and let $K$ be the quotient field of $\mathrm{D}$. Let $\overline{\mathrm{D}}$ be the completion of $\mathrm{D}$. Then $\overline{\mathrm{D}} \cap K=\mathrm{D}$.

Proof. Assume that $a / b(a, b \in \mathbb{D}, b \neq 0)$ is in $\overline{0}$. Then $a$ is in $b \overline{0}$. Since $b \bar{\jmath} \cap \mathfrak{D}=b \mathrm{D}$, we see that $a$ is in $b \mathfrak{D}$, whence $a / b$ is in $\mathfrak{D}$. Thus we see our assertion.

Now we prove

Lemma 13. Let o be a Noetherian local integrity domain. Assume that the completion $\overline{\mathrm{D}}$ of $\mathrm{D}$ has no nilpotent element. Then the integral closure $\mathrm{D}^{\prime}$ of $\mathrm{D}$ in the quotient field $K$ of $\mathrm{D}$ is a finite $\mathrm{D}$-module.

Proof. Assume the contrary. Then we can find an infinite ascending chain of subrings $\mathfrak{D}_{n}$ of $\mathfrak{D}^{\prime}$ which begins from $\mathfrak{D}=\mathfrak{D}_{0}$, such that each $\mathfrak{D}_{n}$ is a finite $\mathfrak{D}$ module. Then as is well known, the completion $\overline{\mathrm{D}}_{n}$ of $\mathrm{D}_{n}$ is imbedded in the total quotient ring of $\overline{\mathrm{D}}$. Since $\overline{\mathrm{D}}_{n}$ are integral over $\overline{\mathrm{D}}$ and since $\overline{\mathrm{D}}$ is a subdirect sum of complete local integrity domains, there exists a number $n$ such that $\overline{\mathfrak{D}}_{n+1}=\overline{\mathfrak{D}}_{n}$ by Proposition 1. Then by Lemma 12, we see that $\mathfrak{D}_{n+1}=\mathfrak{D}_{n}$, which is a contradiction.

\section{$\S 2$. Finitely generated integrity domains}

We say that a local ring $\mathfrak{D}$ is of finitely generated type, if $\mathfrak{b}$ is a ring of quotients of a finitely generated ring (over a prime integrity domain) (with respect

11) Some remarks on local rings II, Memo. Kyoto. 
to its prime ideal).

We say that a local ring 0 is of finitely generated type over its subring $D^{\prime}$ which is a local ring, if there exists a finitely generated subring $b^{\prime \prime}$ over $b^{\prime}$ such that $\mathfrak{D}$ is a ring of quotients of $\mathfrak{D}^{\prime \prime}$ with respect to its prime ideal which lies. over the maximal ideal of $D^{\prime}$.

Now to prove the lemma, which is stated in the introduction, on finitely generated integrity domains, it will be sufficient to show the following

LEMMA 14. If a local integrity domain $\mathfrak{D}$ is of finitely generated type, then the integral closure of $\mathrm{D}$ in its quotient field is a finite $\mathrm{D}$-module.

Before proving this lemma, we must prove some preliminary results:

Lemma 15. Let $k$ be $a$ field and let $x_{1}, \ldots, x_{n}$ be algebraically independent element over $k$. Then 1) every maximal ideal of $k\left[x_{1}, \ldots, x_{n}\right]$ can be generated by $n$ elements and 2) if $\mathfrak{p}$ is a prime ideal of $k\left[x_{1}, \ldots, x_{n}\right]$ then rank $\mathfrak{p}+\operatorname{dim} \mathfrak{p}$. $=n$ and dim $\mathfrak{p}$ is equal to the transcendence degree of $k\left[x_{1}, \ldots, x_{n}\right]_{\mathfrak{p}}$ over $k$

Proof. The first assertion is evident and the second one can be proved easily by Noether's normalization theorem. ${ }^{12)}$

LEMMA 16. Let $\mathfrak{b}$ ba a discrete valuation ring with a prime element $p$ and let $x_{1}, \ldots, x_{n}$ be algebraically independent elements over $\mathfrak{v}$. Then any maximal ideal $\mathrm{m}$ of $\mathfrak{v}\left[x_{1}, \ldots, x_{n}\right]$ which contains $p$ is generated by $n+1$ elements, whence $\mathfrak{v}\left[x_{1}, \ldots, x_{n}\right]_{\mathfrak{m}}$ is a regular local ring of dimension $n+1$.

Proof. Set $k=\mathfrak{b} / p \mathfrak{b}$. Then applying Lemma 16 we see our assertion easily.

Since it is well known that if $D$ is a regular local ring then for any prime idsal $\mathfrak{p}$ of $\mathfrak{o} \operatorname{rank} \mathfrak{p}+\operatorname{dim} \mathfrak{p}=\operatorname{dim} \mathfrak{b},{ }^{13)}$ we see easily the following

Corollary. Let $q$ be a prime ideal of $\mathfrak{v}\left[x_{1}, \ldots, x_{n}\right]$ such that $(q, p)$ does not contain identity. Then $\operatorname{rank} q+\operatorname{dim} q=n+1, \operatorname{dim} q=\operatorname{dim} q \mathfrak{w}\left[x_{1}, \ldots, x_{n}\right]_{\mathfrak{m}}$ for any maximal ideal $\mathrm{m}$ containing $q$ and $p$.

Now since a finitely generated ring over $\mathfrak{v}$ is a homomorphic image of a polynomial ring (in a finite number of indeterminates), it will be easy to see

12) It must be a generalized assertion also for the case of finite field. A proof of such generalization was given in a previous paper 1. c. note 8 ).

13) See, for inst., W. Krull, Dimensionstheorie in Stellenringen, J. Reine Angew. Math. 179 (1938). 
the following assertion by virtue of the corollary to Lemma 16 :

ThEOREM 8. Assume that a local integrity domain $\mathrm{D}$ is of finitely generated type over a discrete valuation ring $\mathfrak{b}$. Let $d$ be the transcendence degree of the residue field of $\mathfrak{b}$ over that of $\mathfrak{b}$. Then the transcendence degree of $\mathfrak{b}$ over $\mathfrak{b}$ is equal to $d+\operatorname{dim} \mathrm{D}-1$.

Remark. If we consider a local integrity domain $\mathfrak{b}$ which is of finitely generated type over a field $k$. then we see easily that the transcendence degree of $\mathfrak{D}$ over $k$ is equal to the sum of the dimension of $\mathfrak{D}$ and the transcendence degree of the residue field of $\mathfrak{o}$ over $k$.

On the other hand, it is easy to find a discrete valuation ring $\mathfrak{v}^{\prime}$, for a local integrity domain $\mathfrak{D}$ which is of finitely generated type over a valuation ring $\mathfrak{b}$, such that 1) $\mathfrak{b}^{\prime}$ is a subring of $\mathfrak{b}$ and 2) $\mathfrak{b}$ is of finitely generated type over $\mathfrak{b}^{\prime}$ and the residue field of $\mathfrak{D}$ is a finite algebraic extension of that of $\mathfrak{b}^{\prime}$ : Such a valuation ring $\mathfrak{b}^{\prime}$ is called a basic ring of $\mathfrak{b}$. Then Theorem 8 shows in particular the following

Proposition 2. Assume that a local integrity domain $\mathfrak{D}$ is of finitely generated type over its basic ring $\mathfrak{v}$ and let $p$ be a prime element of $\mathfrak{v}$. If $p, x_{1}$, $\ldots, x_{n}$ is a system of parameters of $\mathfrak{b}$, then $\mathfrak{o}$ is algebraic over $\mathfrak{b}\left[x_{1}, \ldots, x_{n}\right]$.

Next we consider a discrete valuation ring $\mathfrak{b}$ which has only countably many elements (here we assume that $\mathfrak{b}$ is of $\operatorname{rank} 1$ ). Let $x_{1}, \ldots, x_{n}$ be algebraically independent elements over $\mathfrak{v}$ and let $\mathfrak{v}$ be a local integrity domain which is a ring of quotient of $\mathfrak{b}\left[x_{1}, \ldots, x_{n}\right]$ with respect to a maximal ideal $m$ which contains a prime element $p$ of $\mathfrak{v}$. Then $\mathfrak{D}$ is an integrally closed Noetherian local integrity domain and has only countably many elements. Let $D^{*}$ be the Henselization of $\mathfrak{b}$. Then we can find a sequence of subrings $\mathfrak{b}_{i}$ of $\mathrm{b}^{*}$ such that 1) each $\mathfrak{D}_{i}$ is a finite decompositional extension of $\mathfrak{D}$ with a characteristic prime ideal $\left.\mathfrak{p}_{i}, 2\right) \mathfrak{p}_{\mathfrak{i p}_{i}} \subset \mathfrak{D}_{i+1 \mathfrak{p}_{i+1}}$ for each $i$ and 3$)$ the union of all $\mathfrak{p}_{i p_{i}}$ coincides with $D^{*}$ (existence of such sequence is easy because the algebraic closure of the field of quotients of $\mathfrak{D}$ has countably many elements). Therefore, for this $\mathfrak{D}$, we can prove easily our assertions in the present paper (in Chapters I and II), making no use of the lemma which we want to prove.

By the same reason, we see that our results in the present paper holds good for regular local rings which are of finitely generated type over a finite 
field. ${ }^{11)}$

On the other hand, we can prove

Proposition 3. Let $\mathfrak{r}$ be a Henselian regular local ring and let $m$ be its maximal ideal. Assume that $\mathfrak{r}$ satisfies the following two conditions:

1) If $\mathfrak{p}$ is a minimal prime ideal of $\mathfrak{r}$, then any finite integral extension of $r / p$ is algebraically closed in its completion.

2) Any almost finite integral extension of $\mathfrak{r}$ is a finite $\mathfrak{r}$-module.

Then $\mathfrak{r}$ is algebraically closed in its completion.

And then

Proposition 4. Let $\mathfrak{r}$ be a regular local ring of finitely generated type over a (finite) field. Then the Henselization of $\mathfrak{r}$ is algebraically closed in its completion.

\section{And at last}

Proposition 5. Let $\mathrm{D}$ a local integrity domain which is of finitely generated type. Let $\mathfrak{v}$ be a basic ring of $\mathfrak{D}$. We assume that $\mathfrak{v}$ is not a field but a discrete valuation ring of characteristic zero; let $p$ be a prime element of $\mathfrak{v}$. If $p, x_{1}$, $\ldots, x_{r}$ is a system of parameters of $\mathfrak{v}$, then the Henselization of $\mathfrak{r}=\mathfrak{v}\left[x_{1}, \ldots\right.$, $\left.x_{r}\right]_{\left(p, x_{1}, \ldots, x_{r}\right)}$ is algebraically closed in its completion and for any imbedding of $\mathfrak{b}$ in an algebraic extension of the Henselization $\mathfrak{r}^{*}$ of $\mathfrak{r}, \mathfrak{r}^{*}[\mathfrak{0}]$ is a finite module over $\mathfrak{r}$. And furthermore, the completion of $\mathfrak{D}$ has no nilpotent element.

Proofs of them will be stated in a latter paper (1. c. note 10)).

Now by the last statement in Proposition 5, we see the assertion in Lemma 14, by virtue of Lemma 13.

Added in proof. The writer found a better proof of Lemma 13 than that given here, which will be contained in a latter paper on algebraic geometry over Dedekind domains.

Mathematical Institute, Kyoto University

14) When an integrally closed quasi-local integrity domain $\mathfrak{o}$ contains a field, then considering a finitely generated subring over a subfield of $\mathrm{b}$, we can prove our results in the present paper, also without making no use of the lemma which we want to show now. 